\title{
Application of the Newton-Raphson Method to Lifetime Spectroscopy for Extraction of Defect Parameters
}

\author{
Yan Zhu, Quoc Thong Le Gia, Mattias Klaus Juhl, Gianluca Coletti, and Ziv Hameiri
}

\begin{abstract}
Defect parameter solution surface is a widely accepted method to determine the values of the energy level and the carrier capture cross sections of a defect from injection-dependent lifetime measurements. Results of the defect parameter solution surface come from the recognition of intersection points, which can be difficult when the doping or the temperature range of the measurement is narrow. In this paper, we introduce a new method to extract the defect parameters from lifetime spectroscopy data. After linearization of the Shockley-Read-Hall (SRH) lifetime equation, the problem is transformed into a mathematical form, which can be solved by the Newton-Raphson method. This method requires a much shorter computing time and directly provides the possible values of the defect parameters without user interaction, even for measurements with narrow doping and temperature ranges.
\end{abstract}

Index Terms-Defect, lifetime, Newton-Raphson method, recombination.

\section{INTRODUCTION}

D EFECTS in silicon wafers have a detrimental effect on the performance of solar cells as they increase the ShockleyRead-Hall (SRH) recombination rate and consequently reduce the excess carrier lifetime [1]. The recombination activity of a defect can be characterized by three parameters: its energy level $\left(E_{t}\right)$, the electron capture section $\left(\sigma_{n}\right)$, and the hole capture cross section $\left(\sigma_{p}\right)$. The extraction of such parameters is essential for the identification of different defects and for understanding their recombination properties. Deep-level transient spectroscopy (DLTS) has been widely used for the determination of the defect parameters. In the last few decades, an alternative techniqueinjection-dependent lifetime spectroscopy (IDLS)—has been

Manuscript received January 15, 2017; revised March 2, 2017 and March 28, 2017; accepted April 16, 2017. Date of publication April 24, 2017; date of current version June 19, 2017. This work was supported by the Australian Government through the Australian Renewable Energy Agency under Grant 2014/RND097. The work of Z. Hameiri was supported by the Australian Research Council through the Discovery Early Career Researcher Award under Grant DE150100268. The views expressed herein are not necessarily the views of the Australian Government, and the Australian Government does not accept responsibility for any information or advice contained herein. (Corresponding author: Yan Zhu.)

Y. Zhu, Q. T. Le Gia, M. K. Juhl, and Z. Hameiri are with the University of New South Wales, Kensington, N.S.W. 2052, Australia (e-mail: yan.zhu@student.unsw.edu.au; qlegia@unsw.edu.au; mattias.juhl@ unsw.edu.au; z.hameiri@unsw.edu.au).

G. Coletti is with the University of New South Wales, Kensington, N.S.W. 2052, Australia, also with the Energy Research Centre of the Netherlands, Petten 1755 LE, The Netherlands (e-mail: coletti@ecn.nl).

Color versions of one or more of the figures in this paper are available online at http://ieeexplore.ieee.org.

Digital Object Identifier 10.1109/JPHOTOV.2017.2695666 developed for defect parameterization. Courtesy of its low detection limit and simple sample preparation, IDLS has been extensively used for the characterization of a wide variety of bulk defects, such as iron [2], [3], chromium [4], [5], aluminum [6], [7], copper [8], oxygen precipitates [9], and many more [10].

Currently, IDLS data are analyzed using the defect parameter solution surface (DPSS) method developed by Rein [10]. This method is based on fitting of the measured injection-dependent lifetime data with the SRH model [11], [12]. For each energy level within the bandgap, the two capture cross sections are determined by least squares minimization (usually the minority capture cross section and the capture cross section ratio $k=\sigma_{n} / \sigma_{p}$ are used instead). For a wide range of energy levels, the least square fitting provides exactly the same fitting quality. Therefore, a single IDLS measurement provides a wide range of possible combinations of the three defect parameters, commonly referred to as a DPSS curve, for example, the lines shown in Figs. 1-3 represent combinations of $E_{t}$ and $k$ that have identical fitting quality. To determine the defect parameter explicitly, more IDLS measurements need be taken on samples with different doping concentrations ( $N_{\text {dop }}$-IDLS) or on the same sample at different temperatures [temperature- and IDLS (TIDLS)]. If the DPSS curves for each temperature or doping concentration are plotted together, the true values of the defect parameters are found at the intersection of the different DPSS curves. A detailed description of the DPSS method can be found in the extensive study by Rein [10]. Recently, a different method was used by Sun et al. [4], where the defect parameters were extracted by simultaneously fitting all of the IDLS curves. This method is based on the same principles as the standard DPSS method; however, a more complicated fitting algorithm is required. More recently, Bernardini et al. proposed another alternative method to obtain $E_{t}$ and $k$, referred to as defect parameters contour mapping (DPCM) [13]. The method sweeps through all possible combinations of $E_{t}$ and $k$ and provides the combination with the lowest residuals. DPCM provides a good visualization of the best-fitted defect parameters and potentially their uncertainty range; however, it is time consuming to sweep through the entire range of possible values of defect parameters.

A mathematical method to linearize the IDLS data was proposed by Murphy et al. [14]. This technique was later applied by Morishige et al. for DPSS analysis [15]. The DPSS curves are calculated using the slope and intercept of the linearized IDLS curve. In this method, the fitting process is greatly sim- 
plified, shifting from a relatively complicated fitting of the SRH equation to a straight line fit. However, in order to extract defect parameters using this simplified DPSS method, identification of the intersections between different measurements must still be carried out, either manually or through the use of additional computer algorithms.

This paper demonstrates a new method to extract defect parameters from the linearized IDLS data. Through arranging the problem into a mathematical form consisting of a system of nonlinear equations, the problem can be solved by the NewtonRaphson method [16]. Due to the quadratic convergence nature of the Newton-Raphson method, it is very efficient in finding the defect parameters while providing the same information as the conventional DPSS method. Moreover, similar to the method of Sun et al. [4] and DPCM [13], this method does not require the identification of intersection points of DPSS curves.

\section{METHOD}

\section{A. Rearrangement of the Problem}

For a p-type silicon wafer, if trapping is negligible, the SRH lifetime $\tau_{\mathrm{SRH}}$ can be written as [11], [12]

$$
\tau_{\mathrm{SRH}}=\frac{\tau_{n 0}\left(p_{0}+p_{1}+\Delta n\right)+\tau_{p 0}\left(n_{0}+n_{1}+\Delta n\right)}{p_{0}+n_{0}+\Delta n}
$$

where $\tau_{n 0}\left(\tau_{p 0}\right)$ is the electron (hole) capture time constant, $p_{0}$ $\left(n_{0}\right)$ is the hole (electron) concentration at thermal equilibrium, $p_{1}\left(n_{1}\right)$ is the hole (electron) SRH density, and $\Delta n$ is the excess electron concentration. $\tau_{n 0}$ and $\tau_{p 0}$ are functions of $\sigma_{n}$ and $\sigma_{p}$ :

$$
\begin{aligned}
\tau_{n 0} & =1 /\left(\sigma_{n} v_{\mathrm{th}, e} N_{t}\right) \\
\tau_{p 0} & =1 /\left(\sigma_{p} v_{\mathrm{th}, h} N_{t}\right)
\end{aligned}
$$

where $v_{\mathrm{th}, h}\left(v_{\mathrm{th}, e}\right)$ is the hole (electron) thermal velocity and $N_{t}$ is the concentration of defect. The SRH densities $n_{1}$ and $p_{1}$ are both functions of $E_{t}$ defined as

$$
\begin{aligned}
& n_{1}=n_{i} \exp \left(-\frac{\left(E_{i}-E_{t}\right)}{k_{b} T}\right) \\
& p_{1}=n_{i} \exp \left(-\frac{\left(E_{t}-E_{i}\right)}{k_{b} T}\right)
\end{aligned}
$$

where $n_{i}$ is the intrinsic carrier density, $T$ is the temperature in Kelvin, and $k_{b}$ is the Boltzmann constant.

According to the method by Murphy et al. [14], when the minority carrier concentration is negligible compared to the majority carrier concentration at thermal equilibrium $\left(n_{0} \ll\right.$ $p_{0}$ for a p-type silicon wafer), the SRH lifetime in (1) can be linearized by introducing an independent parameter $X$ :

$$
\tau_{\mathrm{SRH}}=\left[\tau_{n 0}+\frac{\tau_{p 0} n_{1}}{p_{0}}+\frac{\tau_{n 0} p_{1}}{p_{0}}+X\left(\tau_{p 0}-\frac{\tau_{p 0} n_{1}}{p_{0}}-\frac{\tau_{n 0} p_{1}}{p_{0}}\right)\right]
$$

where $X$ is defined as the ratio between the total electron and the total hole concentrations $X=n / p=\left(n_{0}+\Delta n\right) /\left(p_{0}+\right.$ $\Delta n)$. A similar expression can be found for n-type samples. From (2) to (6), it can be clearly seen that the slope $m$ and the intercept $b$ of the linearized IDLS are both functions of the three defect parameters, $E_{t}, \sigma_{n}$, and $\sigma_{p}$ :

$$
\left\{\begin{array}{l}
m=\tau_{p 0}\left(\sigma_{p}\right)-\frac{\tau_{p 0}\left(\sigma_{p}\right) n_{1}\left(E_{t}\right)}{p_{0}}-\frac{\tau_{n 0}\left(\sigma_{n}\right) p_{1}\left(E_{t}\right)}{p_{0}} \\
b=\tau_{n 0}\left(\sigma_{n}\right)+\frac{\tau_{p 0}\left(\sigma_{p}\right) n_{1}\left(E_{t}\right)}{p_{0}}+\frac{\tau_{n 0}\left(\sigma_{n}\right) p_{1}\left(E_{t}\right)}{p_{0}}
\end{array} .\right.
$$

Now, the problem is transformed into the form described by (7). There are three unknown parameters with only two equations; therefore, an infinite number of solutions can be found; this also explains the ambiguity of a single IDLS measurement. To make this problem uniquely solvable, additional information is required. As mentioned before, this can be achieved using $N_{\mathrm{dop}}$-IDLS or TIDLS. When two IDLS measurements are made, four equations are obtained while the unknown parameters remain as three. This problem can be now solved by the Newton-Raphson method.

\section{B. Application of the Newton-Raphson Method}

The Newton-Raphson method [16] is an efficient method to find roots of a function. The root of a function $f(x)=0$ can be found by starting from an initial guess of the root $x_{0}$; an improved approximation of the root can then be calculated by $x_{1}=x_{0}-f\left(x_{0}\right) / f^{\prime}\left(x_{0}\right)$. By repeating this process as

$$
x_{n+1}=x_{n}-f\left(x_{n}\right) / f^{\prime}\left(x_{n}\right)
$$

an accurate result of the root can be obtained very quickly due to the quadratic convergence of this method. Let $x$ in (8) be the vector of the three unknown defect parameters of (7):

$$
x=\left(\begin{array}{c}
\sigma_{n} \\
\sigma_{p} \\
E_{t}
\end{array}\right) .
$$

Let $f(x)$ be the functional form of (7):

$$
f(x)=\left(\begin{array}{l}
f_{m}^{1} \\
f_{b}^{1} \\
f_{m}^{2} \\
f_{b}^{2}
\end{array}\right)=\left(\begin{array}{l}
m^{1}-\left(\tau_{p 0}^{1}-\frac{\tau_{p 0}^{1} n_{1}^{1}}{p_{0}^{1}}-\frac{\tau_{n 0}^{1} p_{1}^{1}}{p_{0}^{1}}\right) \\
b^{1}-\left(\tau_{n 0}^{1}+\frac{\tau_{p 0}^{1} n_{1}^{1}}{p_{0}^{1}}+\frac{\tau_{n 0}^{1} p_{1}^{1}}{p_{0}^{1}}\right) \\
m^{2}-\left(\tau_{p 0}^{2}-\frac{\tau_{p 0}^{2} n_{1}^{2}}{p_{0}^{2}}-\frac{\tau_{n 0}^{2} p_{1}^{2}}{p_{0}^{2}}\right) \\
b^{2}-\left(\tau_{n 0}^{2}+\frac{\tau_{p 0}^{2} n_{1}^{2}}{p_{0}^{2}}+\frac{\tau_{n 0}^{2} p_{1}^{2}}{p_{0}^{2}}\right)
\end{array}\right)
$$

where the superscripts " 1 " and " 2 " indicate measurements at different temperatures or doping levels. $f^{\prime}(x)$ of such a multiple variables problem can be replaced by the Jacobian matrix, which is defined as

$$
J\left(\sigma_{n}, \sigma_{p}, E_{t}\right)=\left(\begin{array}{lll}
\frac{\partial f_{m}^{1}}{\partial \sigma_{n}} & \frac{\partial f_{m}^{1}}{\partial \sigma_{p}} & \frac{\partial f_{m}^{1}}{\partial E_{t}} \\
\frac{\partial f_{b}^{1}}{\partial \sigma_{n}} & \frac{\partial f_{b}^{1}}{\partial \sigma_{p}} & \frac{\partial f_{b}^{1}}{\partial E_{t}} \\
\frac{\partial f_{m}^{2}}{\partial \sigma_{n}} & \frac{\partial f_{m}^{2}}{\partial \sigma_{p}} & \frac{\partial f_{m}^{2}}{\partial E_{t}} \\
\frac{\partial f_{b}^{2}}{\partial \sigma_{n}} & \frac{\partial f_{b}^{2}}{\partial \sigma_{p}} & \frac{\partial f_{b}^{2}}{\partial E_{t}}
\end{array}\right)
$$

while the inverse of the Jacobean matrix can be calculated as $\left(J^{T} J\right)^{-1} J^{T}$. 


\section{Possible Problems and Solutions}

The Newton-Raphson method requires an initial guess of the solution. If the initial guess is not well chosen, several practical problems may arise. When the rank of the Jacobian decreases below the number of variables (here being three), the iteration stops and fails to converge. To ensure this does not plague the result, the rank of the Jacobean is checked in the routine and if it is below three the program restarts with a new randomly chosen initial guess. Another possible issue with this method is that it can converge at an energy level outside the bandgap of the material. To avoid this situation, constraints on the three defect parameters are set according to their physical range. A check has been placed in the routine to assign a random value (within the set physical range) when the parameter departs from the set range, then the iteration can continue and quickly converges to the correct solution.

The application of a physical range on the defect parameters is also used to determine the possible energy level of the defect in the upper or lower half of the bandgap. Due to the symmetricity of the bandgap, two intersections occur in the DPSS; one intersection in the upper half of the bandgap and one in the lower half of the bandgap [10]. Similarly, the proposed Newton-Raphson method can also converge to two possible solutions depending on the initial guess. By restricting the physical range of the defect energy level $\left(E_{t}-E_{i}>0\right.$ or $\left.E_{t}-E_{i}<0\right)$, the method provides the possible solution in the upper half or lower half of the bandgap. Although it cannot provide additional information regarding which bandgap half the defect is located in, the method does ensure that no possible solution is missed. Whether the defect is in the upper or lower bandgap half can then be determined by the method proposed by Murphy et al. [14], Rein [10], Paudyal et al. [17], or other measurement techniques, such as DLTS. The approaches discussed above free the users from the requirement of choosing the initial guess. The method will automatically find the two possible solutions in the two bandgap halves.

\section{VERIFICATION OF THE METHOD}

In order to test the reliability of the proposed method, it is compared to the conventional DPSS method for several sets of simulated data. The method is then applied to a set of experimental data from Sun et al. [4] and the results of both methods are compared. In our simulation, the model for dopant ionization is from Altermatt et al. [18]. The intrinsic carrier concentration is calculated according to models from Couderc et al. [19]. The bandgap and bandgap narrowing models are, respectively, from Passler [20] and Yan and Cuevas [21]. The thermal velocity is calculated from models by Green [22].

\section{A. Verification With Simulated Data}

To demonstrate its accuracy, the Newton-Raphson method and conventional DPSS method are applied to ideal $N_{\text {dop }}$-IDLS as well as TIDLS data modeled according to (1) without any simulated noise. The defect parameters used in these simulations are $E_{t}-E_{i}=-0.33 \mathrm{eV}, \sigma_{n}=2.4 \times 10^{-14} \mathrm{~cm}^{2}, \sigma_{p}=0.8 \times$ $10^{-14} \mathrm{~cm}^{2}$ (capture cross section ratio $k=\sigma_{n} / \sigma_{p}=3$ ), and

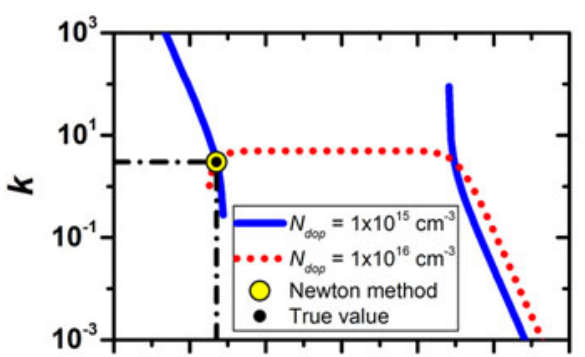

(a)

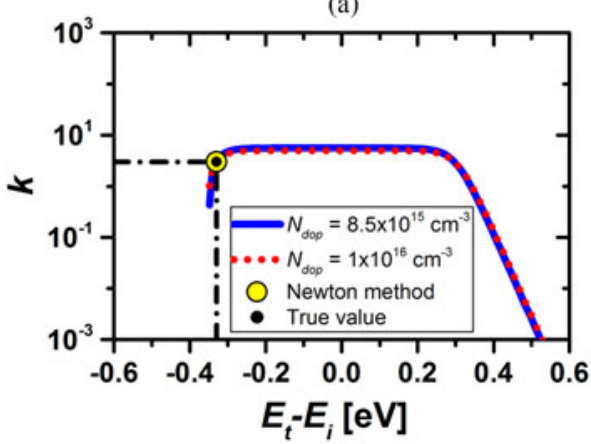

(b)

Fig. 1. Comparison of results from DPSS and Newton-Raphson method for simulated $N_{\text {dop }}$-IDLS of n-type wafer at $300 \mathrm{~K}$ with two doping levels of (a) $1 \times 10^{15}$ and $1 \times 10^{16} \mathrm{~cm}^{-3}$ and (b) $8.5 \times 10^{15}$ and $1 \times 10^{16} \mathrm{~cm}^{-3}$.

$N_{t}=1 \times 10^{12} \mathrm{~cm}^{-3}$. In order to apply these two methods, at least two sets of IDLS data measured at different conditions are needed. Note that more sets of data will increase the reliability of the result. To simplify the demonstration, only two data sets are analyzed in the first simulation. It is also assumed that the defect is in the lower bandgap half; thus, only one solution instead of two for the Newton-Raphson method is presented.

We modeled two sets of $N_{\text {dop }}$-IDLS data with different doping ranges: The first set contains two n-type wafers with doping concentrations of $1 \times 10^{15}$ and $1 \times 10^{16} \mathrm{~cm}^{-3}$, whereas the second set contains two wafers with closer doping levels of $8.5 \times 10^{15}$ and $1 \times 10^{16} \mathrm{~cm}^{-3}$. As presented in Fig. 1, the chosen bulk doping levels of the first set lead to a "split" DPSS$k$ curve (blue solid line) and a "continuous" DPSS curve (red dotted line), whereas the doping levels of the second set lead to two "continuous" DPSS curves that are close to each other. The true energy level and capture cross section ratio $k$ are indicated by the black dashed-dotted lines. In both cases, one of the intersections pinpoints the true defect parameter. However, identification of the intersection points in the second set of simulated data is more difficult as the two DPSS curves are too close to each other in such a small doping range. The results obtained by the Newton-Raphson method are presented with yellow circles. As can be seen, the Newton-Raphson method accurately determines the true defect parameter values (in the range of $<0.1 \%$ ), indifferent of the range of doping levels. Since defect parameter values are directly obtained, there is no need for an intersection identification step. This is one of the advantages of the proposed method.

The computing time of the two methods was also investigated. It was found that the computing time of the Newton-Raphson method is at least $30 \%$ faster than the conventional DPSS 


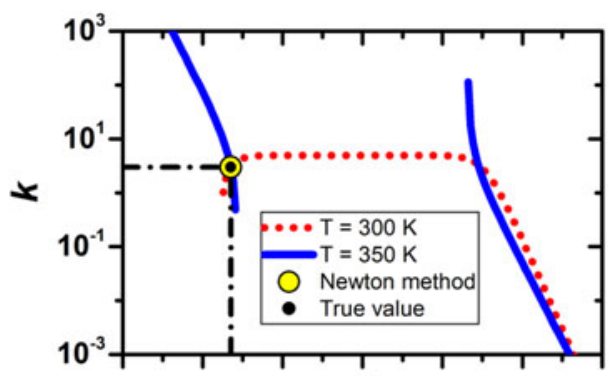

(a)

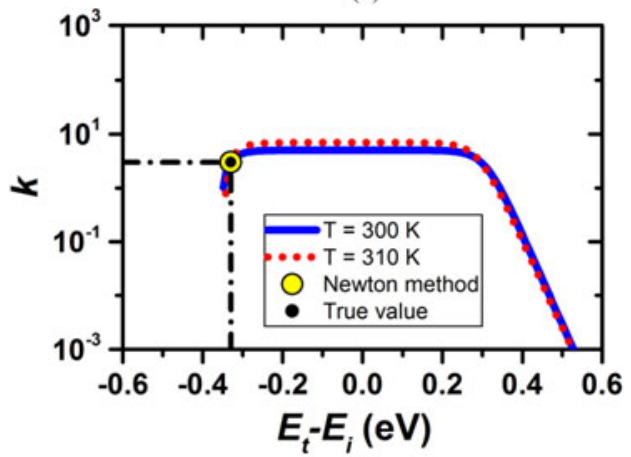

(b)

Fig. 2. Comparison of results from DPSS and Newton-Raphson method for simulated TIDLS for of an n-type wafer with doping concentration of $1 \times$ $10^{16} \mathrm{~cm}^{-3}$ at (a) 300 and $350 \mathrm{~K}$ and (b) 300 and $310 \mathrm{~K}$.

method, not including the additional time for the identification of intersections. The slight difference between the true defect values and the ones obtained by the Newton-Raphson method $(0.1 \%)$ originates from the fact that the linear fitting of (5) neglects the impact of $\Delta n$ on the ionization level [18]. For a typical measurement within an injection level below $1 \times 10^{17} \mathrm{~cm}^{-3}$, this impact can be considered negligible.

Similar results are observed for the two sets of simulated TIDLS data with two different temperature ranges, these results are presented in Fig. 2. The proposed method provides an accurate result of the defect parameters regardless of the temperature range of TIDLS, whereas the intersection points of the conventional DPSS become more difficult to identify when the temperature range is narrow, as shown in Fig. 2(b). The conventional DPSS analysis is unable to determine defect parameters if the capture cross section ratio has a strong temperature dependence. If the temperature range of the measurement is reduced to such a small range that the capture cross section ratio can be considered as constant, then the difficulty of intersection identification in DPSS analysis can increase as the DPSS curves get closer to each other. The fact that the Newton-Raphson method accurately determines the true defect parameters, even with a small temperature range, makes this method suitable for the analysis of defect with temperature-dependent capture cross sections.

To analyze the sensitivity of the Newton-Raphson method to noise, the same sets of $N_{\text {dop }}$-IDLS measurements as Fig. 1 were modeled. This time, random noise (10\%) was added to the simulated lifetime data. The results are displayed in Fig. 3(a) and (b). For the data with a wider doping range, the Newton-Raphson method and DPSS technique provide results close to the input

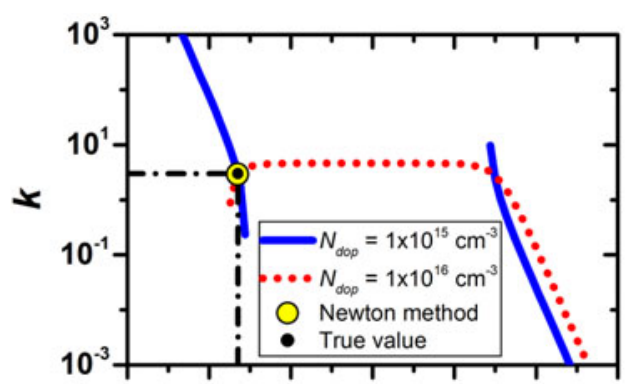

(a)

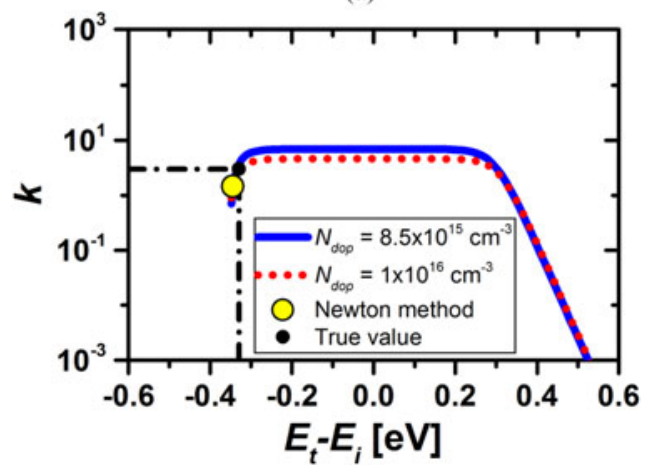

(b)

Fig. 3. Comparison of results from DPSS and Newton-Raphson methods for the same set of simulation as Fig. 1 but with $10 \%$ random noise in the lifetime data.

true parameters; the relative errors of both methods are within $3 \%$. For the simulations presented in Fig. 3(b), which have a narrower doping range, the values determined by the NewtonRaphson method are in good agreement with the intersection point of DPSS, but calculated $E_{t}$ and $k$ from both methods are further from the true defect parameters (4\% error in $E_{t}$ and $52 \%$ error in $k$ ). The simulations indicate that the Newton-Raphson method has similar sensitivity to measurement uncertainty as conventional DPSS curves. This is not surprising as it originates from a rearrangement of the DPSS method. However, it avoids the potential uncertainty during the intersection identification, especially when the DPSS curves are close to each other.

\section{B. Verification With Experimental Data From the Literature}

The proposed Newton-Raphson method is applied to the $N_{\text {dop }}$-IDLS data of Sun et al. [4]. The data set consists of five IDLS measurements on intentionally chromium contaminated n-type wafers, with doping concentrations between $9.1 \times 10^{15}$ and $1.5 \times 10^{16} \mathrm{~cm}^{-3}$. Sun et al. [4] simultaneously fit the five sets of data and found two possible energy levels of interstitial chromium $\left(\mathrm{Cr}_{\mathrm{i}}\right)$ that provide similar fitting residuals. The one in the upper bandgap half with a value of $E_{i}+0.322 \mathrm{eV}$ was found to be close to previously reported values in the literature. Assuming an 11\% uncertainty in the lifetime measurements, $k$ was initially estimated to be between 0.7 and 12.8. By analyzing the monotonically increasing trend of the measured injection-dependent lifetime curves for all the samples, they further reduced the upper limit of $k$ to 3.2. The proposed Newton-Raphson method as well as conventional DPSS 


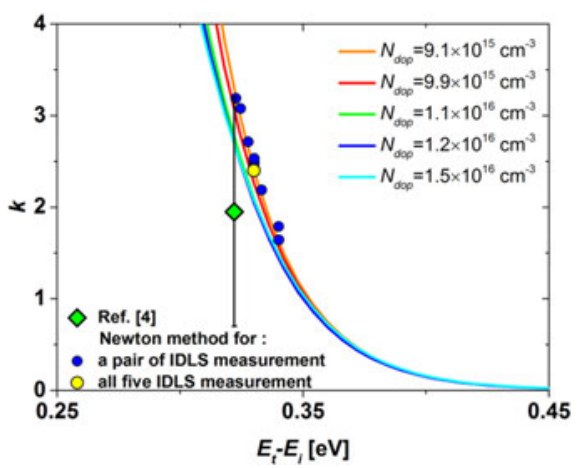

Fig. 4. Comparison of DPSS and the Newton-Raphson method applied to a set of $N_{\mathrm{dop}}$-IDLS data from Sun et al. [4], the other properties of sample at each doping level can be found within this reference.

method is applied to this set of data and the result is presented in Fig. 4. Here, we assume that the bandgap half-information of $\mathrm{Cr}_{i}$ is known from the literature. Due to the small range of the doping levels, it is challenging to identify the intersections of the DPSS curves. This also explains the relatively wide uncertainty range of the reported capture cross section ratio by Sun et al. [4]. The green diamond and associated error bar in Fig. 4 indicate the value range determined by Sun et al. [4], whereas the circles indicate the energy level and capture cross section ratio determined by the Newton-Raphson method. The blue circles are the results of analyzing two different IDLS measurements (five sets of measurements; total of ten combinations), whereas the yellow circle is the result of using all five IDLS measurements. The distribution of the blue circles provides a good indication of the measurement uncertainty as they should overlap with the yellow circle if the measured data are not impacted by noise and measurement-related uncertainties. Therefore, the Newton-Raphson method determined the defect parameter and uncertainty range as $E_{t}-E_{i}=0.33 \pm 0.01 \mathrm{eV}$ and $k=2.4 \pm 0.8$. These values are in good agreement with the values of Sun et al. [4], within the reported range of $k$ and within $2.5 \%$ deviation of the reported $E_{t}$ (note that the uncertainty range of $E_{t}$ was not given in [4]). A possible reason for the deviation could be the error during the digitization of data and the use of different models for parameters such as the thermal velocity.

As mentioned before, the modified method used by Sun et al. [4] determined a relatively large range for $k$ at first. An additional step involving human inspection of the data is required to reduce this range, and this additional step might not be applicable for a different set of data. However, with no human interaction, the proposed Newton-Raphson method extracted the defect parameters, as well as their uncertainty range, in good agreement with the results of Sun et al. [4].

\section{CONCLUSION}

A new method to extract defect parameters from $N_{\text {dop }}$-IDLS or TIDLS measurements is presented. The technique is based on the Newton-Raphson method and has the advantages of short computing time and directly providing the two possible values of defect parameters without need for user interaction, although, additional analysis to determine bandgap half is still required. The reliability of this method is demonstrated through simulations, as well as through analysis of previously published data. An additional important advantage of the new method is the ability to provide relatively accurate values, even when the doping range or the temperature range is too small for a good determination of defect parameters by the conventional DPSS method.

\section{REFERENCES}

[1] G. Coletti, "Sensitivity of state-of-the-art and high efficiency crystalline silicon solar cells to metal impurities," Prog. Photovolt. Res. Appl., vol. 15, pp. 1163-1170, 2012.

[2] B. B. Paudyal, K. R. McIntosh, and D. H. Macdonald, "Temperature dependent electron and hole capture cross sections of iron-contaminated boron-doped silicon," in Proc. 34th IEEE Photovolt. Spec. Conf., 2009, pp. $1588-1593$.

[3] J. E. Birkholz, K. Bothe, D. Macdonald, and J. Schmidt, "Electronic properties of iron-boron pairs in crystalline silicon by temperature-and injection-level-dependent lifetime measurements," J. Appl. Phys., vol. 97, no. 10, 2005, Art. no. 103708.

[4] C. Sun, F. E. Rougieux, and D. Macdonald, "Reassessment of the recombination parameters of chromium in n-and p-type crystalline silicon and chromium-boron pairs in p-type crystalline silicon," J. Appl. Phys., vol. 115, no. 21, 2014, Art. no. 214907.

[5] J. Schmidt, R. Krain, K. Bothe, G. Pensl, and S. Beljakowa, "Recombination activity of interstitial chromium and chromium-boron pairs in silicon," J. Appl. Phys., vol. 102, no. 12, 2007, Art. no. 123701.

[6] C. Sun, F. E. Rougieux, J. Degoulange, R. Einhaus, and D. Macdonald, "Reassessment of the recombination properties of aluminium-oxygen complexes in n- and p-type Czochralski-grown silicon," Phys. Status Solidi, vol. 253, no. 10, pp. 2079-2084, 2016.

[7] J. Schmidt, "Temperature-and injection-dependent lifetime spectroscopy for the characterization of defect centers in semiconductors," Appl. Phys. Lett., vol. 82, no. 13, pp. 2178-2180, 2003.

[8] A. Inglese, J. Lindroos, H. Vahlman, and H. Savin, "Recombination activity of light-activated copper defects in p-type silicon studied by injection- and temperature-dependent lifetime spectroscopy," J. Appl. Phys., vol. 120, no. 12, 2016, Art. no. 125703.

[9] J. D. Murphy, R. E. McGuire, K. Bothe, V. V. Voronkov, and R. J. Falster, "Minority carrier lifetime in silicon photovoltaics: The effect of oxygen precipitation," Sol. Energy Mater. Sol. Cells, vol. 120, pp. 402-411, 2014.

[10] S. Rein, Lifetime Spectroscopy: A Method of Defect Characterization in Silicon for Photovoltaic Applications, vol. 85. New York, NY, USA: Springer, 2006.

[11] W. Shockley and W. T. Read, "Statistics of the recombination of holes and electrons," Phys. Rev., vol. 87, no. 46, pp. 835-842, 1952.

[12] R. N. Hall, "Electron-hole recombination in germanium," Phys. Rev., vol. 87, no. 2, pp. 387-387, 1952.

[13] S. Bernardini, T. U. Naerland, A. L. Blum, G. Coletti, and M. I. Bertoni, "Unraveling bulk defects in high-quality c-Si material via TIDLS," Prog. Photovolt. Res. Appl., vol. 25, no. 3, pp. 209-217, 2017.

[14] J. D. Murphy, K. Bothe, R. Krain, V. V Voronkov, and R. J. Falster, "Parameterisation of injection-dependent lifetime measurements in semiconductors in terms of Shockley-Read-Hall statistics: An application to oxide precipitates in silicon," J. Appl. Phys., vol. 111, no. 11, 2012, Art. no. 113709.

[15] A. E. Morishige et al., "Lifetime spectroscopy investigation of lightinduced degradation in p-type multicrystalline silicon PERC," IEEE $J$. Photovolt., vol. 6, no. 6, pp. 1466-1472, Nov. 2016.

[16] J. Raphson, Analysis Aequationum Universalis. London, U.K.: Typis Tho. Braddyll, 1702, 2009.

[17] B. B. Paudyal, K. R. McIntosh, and D. H. Macdonald, "Temperature dependent carrier lifetime studies on Ti-doped multicrystalline silicon," $J$. Appl. Phys., vol. 105, no. 12, 2009, Art. no. 124510. 
[18] P. P. Altermatt, A. Schenk, B. Schmithüsen, and G. Heiser, "A simulation model for the density of states and for incomplete ionization in crystalline silicon. II. Investigation of Si:As and Si:B and usage in device simulation," J. Appl. Phys., vol. 100, no. 11, 2006, Art. no. 113715.

[19] R. Couderc, M. Amara, and M. Lemiti, "Reassessment of the intrinsic carrier density temperature dependence in crystalline silicon," J. Appl. Phys., vol. 115, no. 9, 2014, Art. no. 93705.

[20] R. Pässler, "Dispersion-related description of temperature dependencies of band gaps in semiconductors," Phys. Rev. B, vol. 66, no. 8, 2002, Art. no. 85201 .
[21] D. Yan and A. Cuevas, "Empirical determination of the energy band gap narrowing in $\mathrm{p}+$ silicon heavily doped with boron," J. Appl. Phys., vol. 116, no. 19, 2014, Art. no. 194505

[22] M. A. Green, "Intrinsic concentration, effective densities of states, and effective mass in silicon," J. Appl. Phys., vol. 67, no. 6, pp. 2944-2954, 1990.

Authors' photographs and biographies not available at the time of publication. 